\title{
TRIB3 suppresses proliferation and invasion and promotes apoptosis of endometrial cancer cells by regulating the AKT signaling pathway
}

This article was published in the following Dove Medical Press journal: OncoTargets and Therapy

\author{
Junjie Qu* \\ Binya Liu* \\ Bilan Li \\ Guiqiang Du \\ Yiran Li \\ Jingyun Wang \\ Laman $\mathrm{He}$ \\ Xiaoping Wan
}

Department of Gynaecology, Shanghai First Maternity and Infant Hospital, Tongji University, Shanghai 20I204, China

*These authors contributed equally to this work
Correspondence: Xiaoping Wan Department of Gynaecology, Shanghai First Maternity and Infant Hospital, Tongii University, Outpatient Building, Gaoke Xi Road, Pudong New District, Shanghai 201204, China

Tel +86 2l 2026 I000

Email wanxiaoping22@।63.com
Objective: The aim of this study was to examine the effect of TRIB3 on proliferation, apoptosis, and migration of endometrial cancer (EC) cells and explore the relationship between TRIB3 and AKT signaling pathway in EC progression.

Methods: Immunohistochemical analysis was performed to measure the expression level of TRIB3 in normal endometrium tissues and EC tissues. Overexpression and shRNA knockdown techniques were applied by transfecting EC cells (ISK and AN3CA), and the effect of TRIB3 on EC cell biological behaviors was evaluated. Cell Counting Kit- 8 and colony formation assays were utilized to investigate EC cell proliferation ability, and flow cytometry was performed to assess the apoptosis of EC cells. Moreover, the migration and invasion of EC cells were detected by transwell assay, and the levels of MMP-2 and MMP-9 were measured by ELISA. Additionally, Western blot analysis was carried out to determine the levels of AKT and p-AKT. Results: The expression level of TRIB3 was higher in EC than normal endometrium tissues, and its overexpression promoted apoptosis and suppressed proliferation of EC cells. Furthermore, TRIB3 retarded the migration and invasion of EC cells and decreased the levels of MMP-2 and MMP-9. Conversely, TRIB3 inhibition enhanced the expression levels of MMP-2 and MMP-9, and proliferation and migration of EC cells but suppressed their apoptosis. Similarly, TRIB3 overexpression reduced while its knockdown increased the level of p-AKT.

Conclusion: TRIB3 inhibited proliferation and migration and promoted apoptosis of EC cells probably through regulating AKT signaling pathway.

Keywords: endometrial carcinomas, Drosophila tribbles homolog 3, endometrium, shRNA knockdown, overexpression

\section{Introduction}

Endometrial cancer (EC) is one of the common malignant genital tumors in female characterized by high recurrence and adverse outcomes. Its morbidity and mortality have presented an ascending trend in the last few years, and it is reported that it occurs over a wide range of age groups from young individuals to elderly women. ${ }^{1,2}$ Surgery, chemotherapy, and radiotherapy have been considered as effective treatments for EC..$^{3,4}$ Alternatively, an increasing number of investigators have focused more attention on exploring and elaborating the pathogenic mechanisms of development and progression of EC. ${ }^{5}$ Remarkably, extensive studies have demonstrated that endoplasmic reticulum (ER) stress is preferentially responsible for EC cell survival, which is induced and accompanied by inflammatory stimuli, hypoxia, and glucose starvation occurring during carcinogenesis. ${ }^{6}$ Ulianich and Insabato ${ }^{7}$ stated that unfolded protein response and GRP78 were activated under the ER stress and subsequently participated in regulating 
the growth and migration of EC cells. Also, previous studies implied that ER stress could regulate the expression level of TRIB3, which is a pseudokinase present in mammals and also known as TRB3, NIPK, SKIP3, and SINK, to control cell proliferation, apoptosis, migration, and invasion..$^{8-10}$

Encouragingly, overwhelming evidence indicated that TRIB3 is closely associated with underlying molecular mechanisms of a wide variety of cancers. ${ }^{11,12} \mathrm{Su}$ et $\mathrm{al}^{13}$ suggested that activated ER stress and altered expression levels of TRIB3 were implicated in the apoptosis of lung cancer cells. Zhou et al ${ }^{14}$ examined the relationship between TRIB3 and lung cancer development and found that TRIB3 was probably associated with cancer occurrence and cell migration through the regulation of JAG1/ Notch pathway.

Additionally, AKT signaling pathway was also found to play an essential role in various cancers, ${ }^{15,16}$ and more interestingly, TRIB3 could target AKT to inhibit the tumorigenesis. ${ }^{12}$ Restelli et al ${ }^{17}$ pointed out that TRIB3 controlled cancer cell processes such as cell proliferation and growth by binding to Ser473 of the AKT protein kinase. Zhang et $\mathrm{al}^{18}$ claimed that TRIB3 overexpression blocked AKT activation and promoted the apoptosis of tongue squamous cell carcinoma. Unfortunately, the effect of TRIB3 on oncogenesis and progression of EC has not been understood. Our study used overexpression and shRNA knockdown techniques to investigate the biological function of TRIB3 in EC cells. The objectives of this work were as follows: to investigate the effect of TRIB3 expression on the development of EC; to examine the impact of TRIB3 on EC cell proliferation, apoptosis, and invasion; and to explore the potential molecular mechanism involved in the progression of EC. The findings of this study may facilitate the development of potential strategies for EC treatment. ${ }^{19}$

\section{Materials and methods}

\section{Cell lines, antibodies, and plasmids}

The human EC cell lines ISHIKAWA (ISK) and AN3CA were purchased from American Type Culture Collection (ATCC) and were cultured in DMEM supplemented with 10\% FBS and maintained in a humidified incubator with $5 \% \mathrm{CO}_{2}$ at $37^{\circ} \mathrm{C}$. Following incubation, they were detached by digestion with $0.25 \%$ trypsin and continually passaged. Tissue samples were collected from hysteromyoma patients who were undergoing hysterectomy and patients suffering from EC between 2014 and 2016. In addition, TRIB3 cDNA overexpression plasmids and TRIB3-shRNA plasmids were obtained from GeneChem Company (Shanghai, China). EC cell lines were transfected with TRIB3 overexpression plasmids or TRIB3shRNA plasmids using Lipofectamine 2000 (Thermo Fisher Scientific, Waltham, MA, USA) in light of the manufacturer's protocols, and subsequently, the transfection efficiency was evaluated. The cell lines were divided into normal control group (NC), TRIB3-shRNA inhibitor negative control group (TINC), TRIB3 overexpression group (T), and TRIB3 inhibitor group (TI). This study was reviewed and approved by the Ethics Committee of Shanghai First Maternity and Infant Hospital, and all subjects gave written informed consent in accordance with the Declaration of Helsinki.

\section{Immunohistochemical analysis of TRIB3 expression}

A tissue microarray (UT801a; Alenabio Company, Xi'an, China), including 13 normal endometrial tissues, five endometritis tissues, 23 endometrial hyperplasia tissues, as well as 25 endometrioid adenocarcinoma tissues, was employed for immunohistochemical staining to examine the expression of TRIB3. The tissue microarray was washed three times for 5 minutes with PBS and then incubated in PBS solution with $3 \% \mathrm{H}_{2} \mathrm{O}_{2}$ to eliminate endogenous peroxidase. This was followed by cleaning with PBS two times for 5 minutes and soaking in PBS containing 1\% normal goat serum for 20 minutes to block the nonspecific binding. Afterwards, TRIB3 primary antibody (1:200; Proteintech Group, Rosemont, IL, USA) was added and incubated at $4^{\circ} \mathrm{C}$ overnight. After washing three times for 5 minutes, secondary antibody marked by horseradish peroxidase (1:500; Dako, Glostrup, Denmark) was added and incubated for 30 minutes at room temperature. Finally, the products were stained with 3,3'-diaminobenzidine, counterstained with hematoxylin, sealed, and observed under a fluorescence microscope (Nikon Eclipse, Tokyo, Japan). Scoring systems were employed to assess the staining intensity of positive cells (no staining: 0; pale brown staining: 1; and brown staining: 2 ) and to identify the percentage of positive cells (unstained cells: 0 ; $\leq 25 \%$ : $1 ; 26 \%-50 \%: 2 ; \geq 50 \%$ : 3 ; the score between 0 and 2 indicates negative and $>2$ indicates positive).

\section{Cell viability assay}

Cell viability of the experimental groups (NC group, TINC group, $\mathrm{T}$ group, and TI group) was examined by Cell Counting Kit-8 (CCK-8) assay. Briefly, cells in logarithmic growth phase were seeded into 96-well plates at a density of $3 \times 10^{4} / \mathrm{mL}$ at a volume of $100 \mu \mathrm{L} /$ well and incubated to support cell attachment. After culturing for 0,48 , and 96 hours, 
CCK-8 solution (10 $\mu \mathrm{L} /$ well) was added into the 96 -well plates, respectively, and cells were maintained and cultured at $37^{\circ} \mathrm{C}$ for 1 hour. Then, the absorbance at $450 \mathrm{~nm}$ was measured with an ELISA reader (Thermo Fisher Scientific, Waltham, MA, USA) to further determine the cell viability. This assay was conducted in triplicate for each group.

\section{Plate clone formation assay}

The ISK and AN3CA cells in logarithmic growth phase were digested using trypsin solution, counted with cellcount boards, and seeded at a density of 100 cells/well in six-well plates. The cells were cultured for 7 days, and the cell growth conditions were observed daily. The cell clones formed were washed twice using PBS solution and fixed with $100 \%$ methanol for 15 minutes followed by drying at room temperature. Then, these cells were stained with Giemsa solution for 30 minutes at room temperature, rinsed three times, and photographed after drying.

\section{AnnexinV-FITC/propidium iodide (PI) apoptosis assay}

The cell apoptosis in all the four groups mentioned above was evaluated by the AnnexinV-fluorescein isothiocyanate (FITC)/PI double-staining method. Cells were plated at a density of $1 \times 10^{6} / \mathrm{mL}$ in serum-free medium and subsequently harvested in the reaction tubes followed by washing twice with PBS. Then, the supernatant was discarded and cells were resuspended in precooled binding buffer $(100 \mu \mathrm{L})$. Afterwards, AnnexinV-FITC $(5 \mu \mathrm{L})$ and PI $(10 \mu \mathrm{L})$ were added to cells, vortexed, and incubated for 15 minutes at room temperature away from light. Finally, the cells were mixed with binding buffer ( $400 \mu \mathrm{L})$ and quantitatively analyzed using a flow cytometer system (BD, Franklin Lakes, NJ, USA).

\section{Transwell assay}

The transwell assay was utilized to determine the migratory abilities of cells in the different treatment groups. The bottom chamber of the transwell was coated with complete medium $(800 \mu \mathrm{L})$. Meanwhile, approximately $4 \times 10^{4}$ cells were suspended in the serum-free medium $(200 \mu \mathrm{L})$ and then seeded onto the upper chamber of the transwell $(8 \mu \mathrm{m}$ pore size) and incubated for 16 hours. Following incubation, the migrated cells adhered to the lower surface of the membranes were stained with calcein-AM $(0.2 \mu \mathrm{g} / \mathrm{mL}$; Thermo Fisher Scientific) for 30 minutes, counted, and visualized. All experiments were carried out at least three times.

\section{ELISA}

TRIB3 level in TRIB3 overexpression groups, TRIB3 interference groups, and corresponding control groups was measured with a commercial ELISA kit (Boster Biological Technology Co. Ltd, Pleasanton, CA, USA). First, the standard solution was added into a 96-well plate and sealed and incubated for 90 minutes at $37^{\circ} \mathrm{C}$. Then, the prepared anti-biotin human MMP-2 antibody and anti-biotin human MMP-9 antibody were separately added and incubated for 60 minutes at $37^{\circ} \mathrm{C}$ followed by the addition of $\mathrm{ABC}$ solution. Subsequently, 3,3',5,5'-tetramethylbenzidine solution was used as chromogenic reagent and the absorbance at $450 \mathrm{~nm}$ was measured after the termination of chromogenic reaction. Finally, the relevant concentration was calculated and standard curves were obtained.

\section{Western blot analysis}

The total protein was separately extracted from cells transfected with the TRIB3 overexpression plasmid and TRIB3 interference plasmid and subjected to SDS-PAGE. Then, the protein was transferred onto nitrocellulose membranes (Millipore), blocked with 5\% nonfat milk, and maintained for 60 minutes at room temperature. Following this, primary antibodies, which included anti-actin (1:2,000; Proteintech Group, Rosemont, IL, USA), anti-TRIB3 (1:1,000; Thermo Fisher Scientific, Waltham, MA, USA), anti-AKT (1:1,000; CST, Danvers, MA, USA), and anti-p-AKT (1:1,000; CST), were added and incubated overnight. Membranes were subsequently washed three times and incubated with secondary antibodies for 60 minutes at room temperature. Finally, the signal from protein bands was visualized by enhanced chemiluminescence reagents (Thermo Fisher Scientific).

\section{Statistical analysis}

The SPSS software (IBM, Armonk, NY, USA) was applied to conduct all the statistical computations in this study, and all data are expressed as mean \pm SD. The significance of differences between two groups was determined by Student's $t$-test, while one-way ANOVA was applied for comparison among more than two groups. $P<0.05$ was considered as significant statistical difference.

\section{Results \\ TRIB3 was highly expressed in endometritis, endometrial hyperplasia, and endometrioid adenocarcinoma}

The TRIB3 expression levels in various endometrial tissues, including normal endometrium, endometritis tissues, 
endometrial hyperplasia tissues, and endometrioid adenocarcinoma tissues (IB, II, and III stages), were investigated by immunohistochemical analysis. The results showed that TRIB3 expression was positive in normal endometrium tissues and was primarily found in the cytoplasm (Figure 1A). Meanwhile, the expression of TRIB3 was dramatically increased in sections of tissues with endometritis, endometrial hyperplasia, and endometrial adenocarcinoma and also detected in the cytoplasm (Figure 1A). Furthermore, the subsequent statistical analysis revealed that TRIB3 expression levels were significantly different between normal endometrium and endometrial adenocarcinoma tissues $(P<0.01)$ (Figure 1B). These findings suggested that the level of TRIB3 was prominently enhanced in EC cells compared with normal tissues.

\section{Assessment of the effects of TRIB3 on the proliferation and clone formation ability of EC cells}

To detect the effects of TRIB3 on proliferation and colony formation capacity, the ISK and AN3CA cells were first transfected with TRIB3 overexpression plasmid and corresponding TRIB3-shRNA interference plasmid, respectively (Figures 2 and 3). Then, the ability of TRIB3 overexpression plasmid to upregulate TRIB3 and the ability of two different shRNA-containing plasmids (TI-1 and TI-2) to downregulate TRIB3 in ISK and AN3CA cells were determined by Western blot analysis (Figure 2A). CCK- 8 assay showed that the overexpression of TRIB3 suppressed cell survival while the inhibition of TRIB3 presented an opposite pattern (Figure 2B-E). Similarly, the plate colony formation assay also indicated that the number of cells in case of high TRIB3 expression was significantly decreased in the transfected group compared to the normal control group, and inversely, larger and more clones were observed in cells with TRIB3 inhibition (Figure 3A and C). Moreover, there were statistically significant differences between control cells and transfected cells in cell proliferation and clone formation ability as shown in Figure 3B and $\mathrm{D}(P<0.05)$.

\section{Detection of the effect of TRIB3 expression on the apoptosis of EC cells}

To investigate whether TRIB3 expression was correlated with the apoptotic effect of EC cells, ISK and AN3CA cells were transfected with TRIB3 overexpression plasmid

A

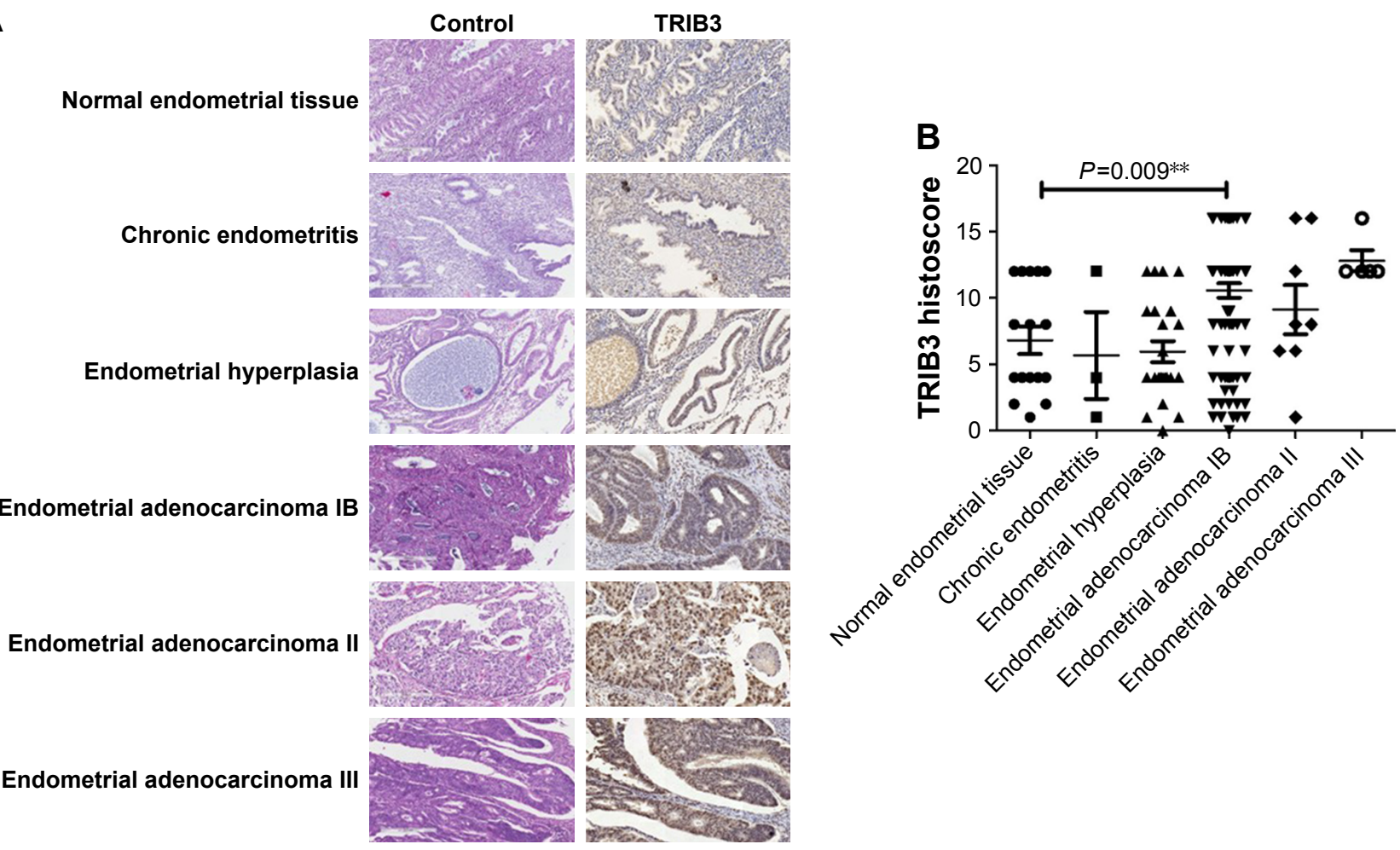

Figure I Immunohistochemical analysis of the expression of TRIB3 in control tissue and tissues with endometritis, endometrial hyperplasia, and endometrioid adenocarcinoma (stages IB, II, and III).

Notes: (A) Representative examples of TRIB3 staining. TRIB3 staining was mainly localized in the cytoplasm. (B) Quantitation of immunohistochemical data. **P $<0.0$ I by ANOVA. 
A
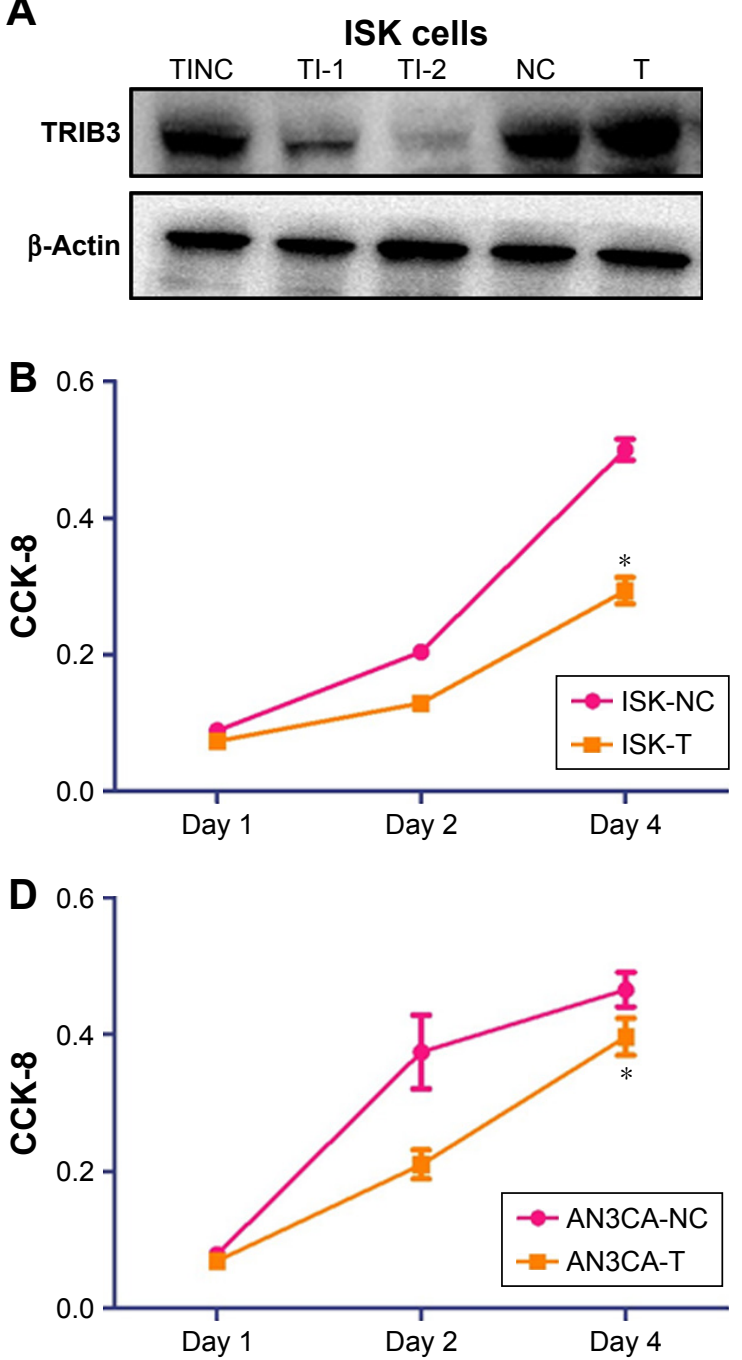

ANC3CA cells

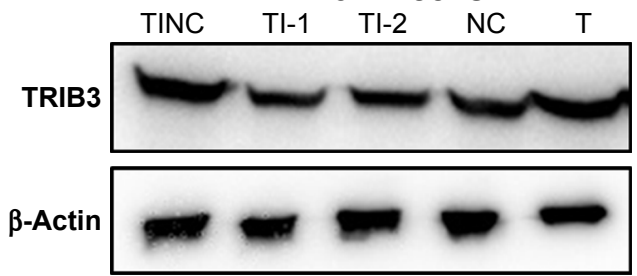

C 0.5

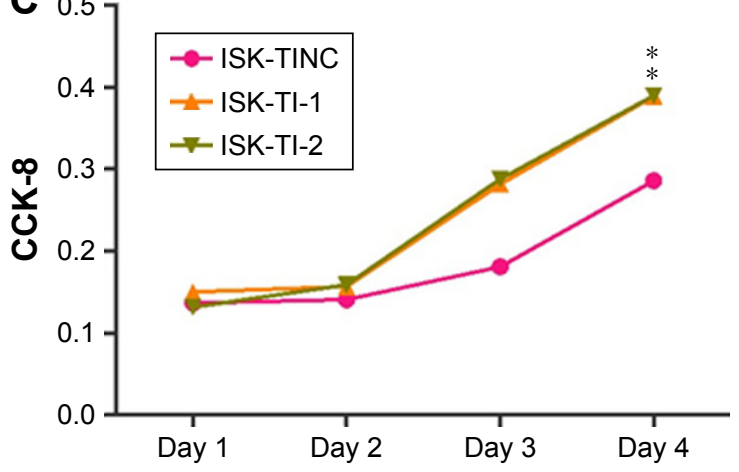

E

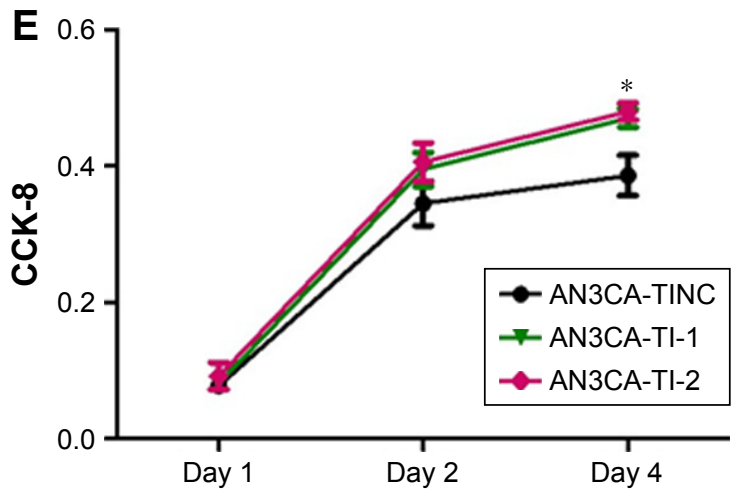

Figure 2 The effect of TRIB3 on ISK and AN3CA cell growth was evaluated by a CCK-8 assay.

Notes: (A) Western blot analysis showed the ability of TRIB3 overexpression plasmid to upregulate TRIB3 and the ability of TRIB3-shRNA plasmid to downregulate the expression of TRIB3 in ISK and AN3CA cells. ISK and AN3CA cells were transfected with (B and D) TRIB3 overexpression plasmids and (C and E) TRIB3-shRNA plasmids. After I, 2, and 4 days, the absorbance of cells was measured by CCK-8. NC: TRIB3 overexpression negative control group; T: TRIB3 overexpression group; TI-I: TRIB3shRNA plasmid I group; TI-2: TRIB3-shRNA plasmid 2 group; TINC: TRIB3-shRNA inhibitor negative control group. $* P<0.05$ and $* * P<0.0$ I, compared with the respective control groups by $t$-test. Data were obtained from three independent experiments.

and TRIB3-shRNA plasmid, respectively. Flow cytometry analysis demonstrated that TRIB3 dramatically enhanced cell apoptosis levels (Figure 4A and C) while the frequency of apoptosis in the TRIB3-shRNA-transfected group was significantly decreased compared with the control group $(P<0.05)$ (Figure 4B and D).

\section{Evaluation of the effect of TRIB3 expression on the migration of EC cells}

The results from transwell assay revealed that TRIB3 overexpression significantly reduced the migratory ability of ISK and AN3CA cells compared with the control group. However, we found that the migration rates in TRIB3 knockdown groups were higher than that of control group
(Figure 5A). Meanwhile, statistical analysis also showed that the effect of TRIB3 on migration of ISK and AN3CA cells was significantly different between experimental groups and control groups (Figure 5B). Additionally, the expression levels of MMP-2 and MMP-9, which were closely associated with cancer cell migration, ${ }^{20}$ were measured to further explore the underlying mechanism of the migration inhibition of EC cells. The results indicated that the expression levels of MMP-2 and MPP-9 were decreased in TRIB3 overexpression groups but enhanced in TRIB3 knockdown groups compared to control groups. Notably, there were significant statistical differences in the levels of MMP-2 and MMP-9 at 72 hours between experimental and control groups (Figure 5C and D). 

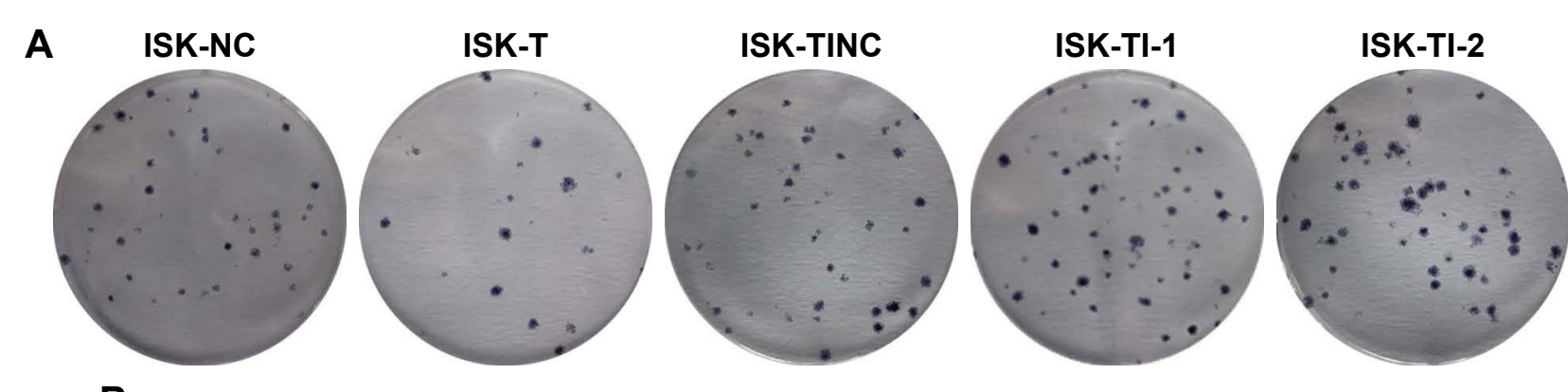

B
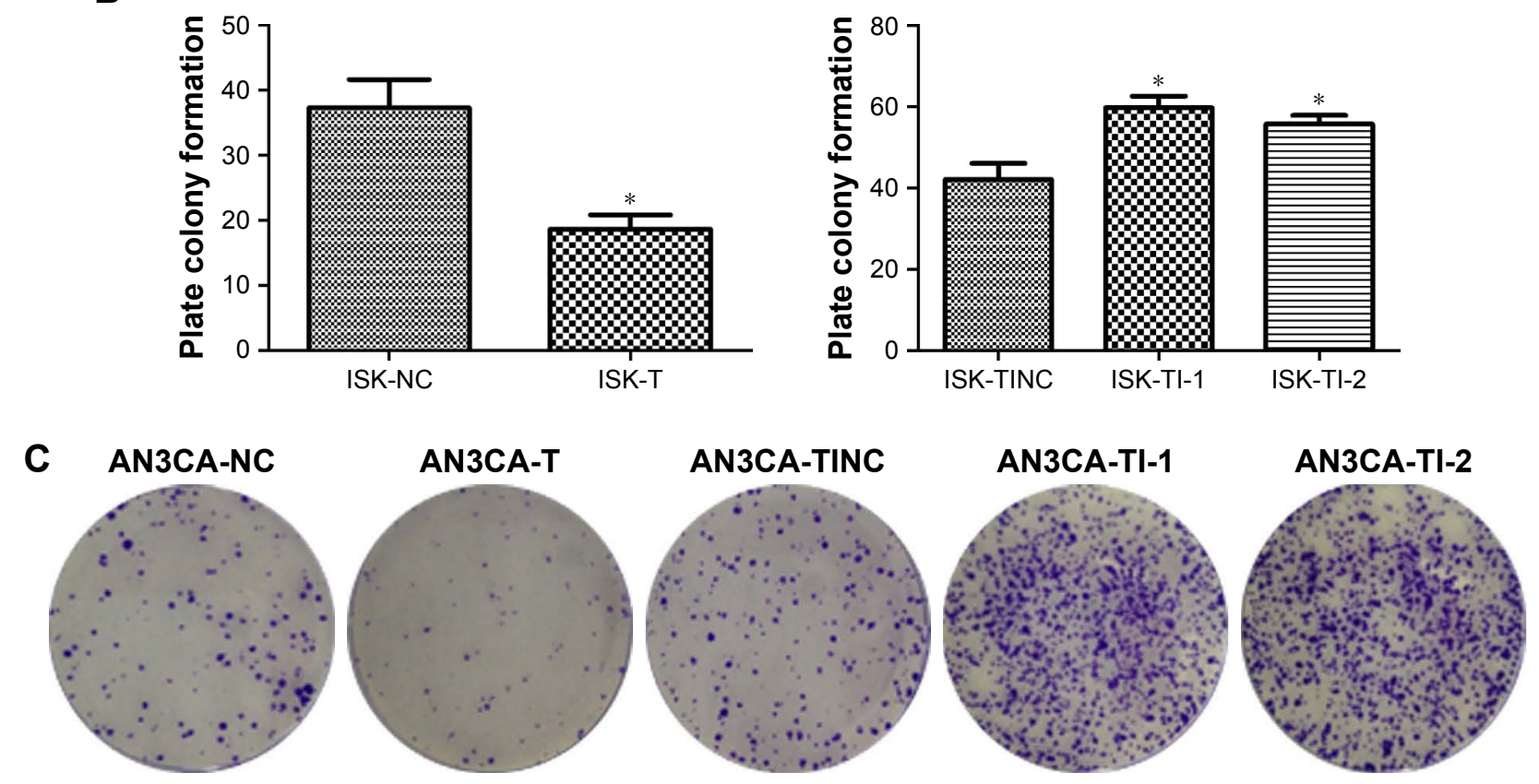

D
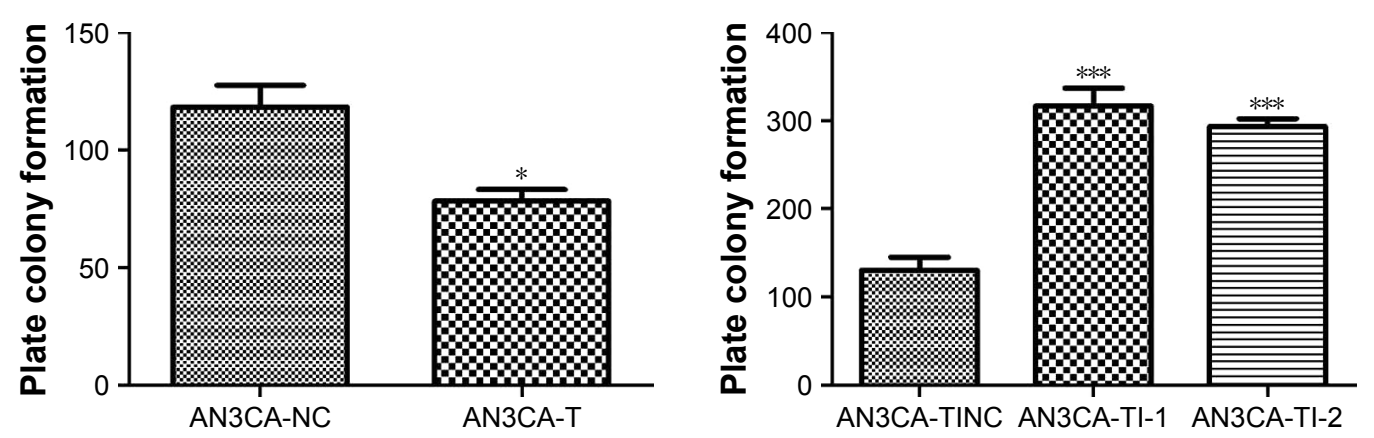

Figure 3 The effect of TRIB3 on ISK and AN3CA cell proliferation was evaluated by the plate colony formation assay.

Notes: The clone formation ability of (A) ISK and (C) AN3CA cells were examined, and the results of statistical analyses of the plate colony formation ability of (B) ISK and (D) AN3CA cells are presented. NC: TRIB3 overexpression negative control group; T: TRIB3 overexpression group; TI-I: TRIB3-shRNA plasmid I group; TI-2: TRIB3-shRNA plasmid 2 group; TINC: TRIB3-shRNA inhibitor negative control group. $* P<0.05$, and $* * * P<0.00$, compared with the respective control groups by $t$-test. Data were obtained from three independent experiments.

\section{Assessment of the correlation between TRIB3 and AKT signaling pathway}

To elucidate the mechanism of TRIB3 involvement, we examined the levels of AKT and p-AKT in the two experimental groups (TRIB3 overexpression and TRIB3 inhibition) of ISK and AN3CA cells, respectively. In TRIB3 overexpression group of ISK cells, the total AKT levels showed no obvious difference compared with control group and there was a marked reduction in AKT phosphorylation levels compared with the control group. Conversely, TRIB3 inhibition experimental group exhibited a significant increase in the AKT phosphorylation levels compared with the control group (Figure 6A). However, there was an apparent decrease in AKT phosphorylation in the TRIB3-shRNA inhibitor negative control group compared to the normal ISK cell group, which may have been caused by protein loading error 

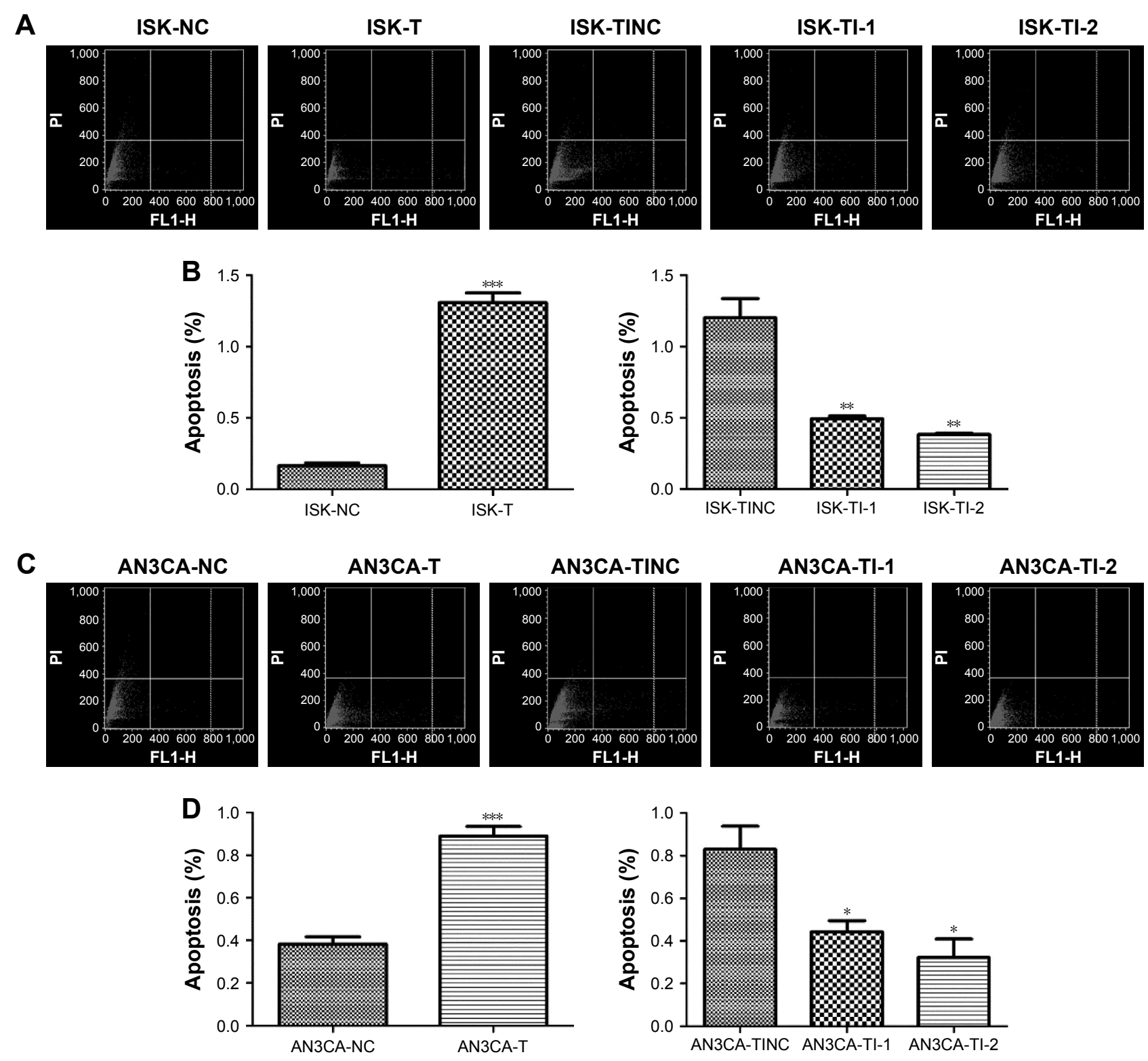

Figure 4 Effects of TRIB3 on apoptosis of ISK and AN3CA cells.

Notes: Contour diagrams for ISK and AN3CA cells are shown in (A and $\mathbf{C})$, respectively and the results of corresponding statistical analyses are displayed in (B and $\mathbf{D})$, respectively. There was a significant difference between normal group and experimental groups (TRIB3 overexpression or TRIB3-shRNA inhibition) of ISK and AN3CA cells. NC: TRIB3 overexpression negative control group; T: TRIB3 overexpression group; TI-I: TRIB3-shRNA plasmid I group; TI-2: TRIB3-shRNA plasmid 2 group; TINC: TRIB3-shRNA inhibitor negative control group. $* P<0.05$, $* * P<0.01$, and $* * * P<0.001$, compared with the respective control groups by $t$-test. Data were obtained from three independent experiments.

(Figure 6A). In addition, a similar trend was observed for the changes in p-AKT levels induced by TRIB3 overexpression and TRIB3 knockdown in AN3CA cells (Figure 6B). Taken together, these results indicated that TRIB3 inhibited AKT activation.

\section{Discussion}

EC is considered as one of the most frequent gynecological malignancies characterized by high incidence and poor prognosis. ${ }^{21}$ Numerous researchers have concentrated on exploring the underlying pathogenesis mechanism of EC in the last few years. ${ }^{22} \mathrm{~A}$ wealth of evidence has demonstrated that a wide variety of molecules such as miRNAs and genes are probably associated with EC. ${ }^{23-25}$ Remarkably, TRIB3 has recently been reported to be implicated in multiple cancers including tongue squamous cell carcinoma, gastric cancer, and breast cancer. ${ }^{18,26,27}$ In the present study, the findings revealed that TRIB3 expression levels were enhanced in endometrioid adenocarcinoma compared with the normal tissues, and TRIB3 could promote apoptosis and inhibit proliferation and colony formation of EC cells and possibly plays important roles in the AKT signaling pathway. 


\section{A}
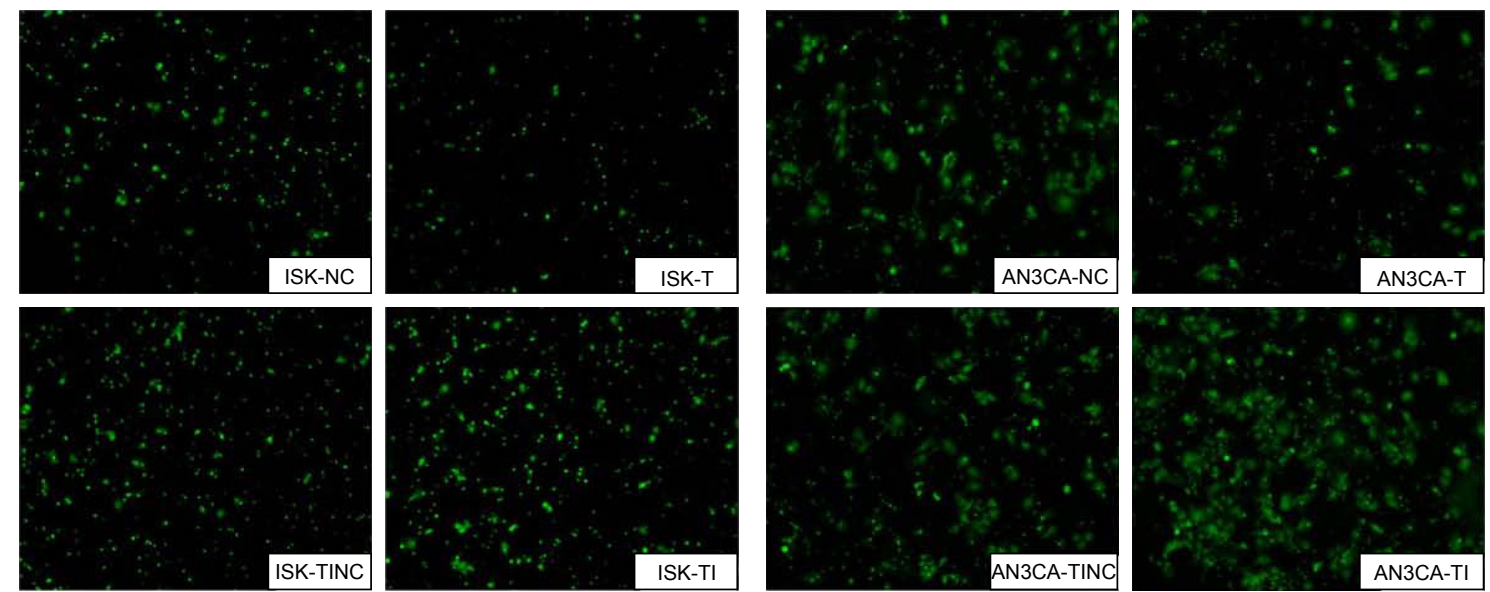

B
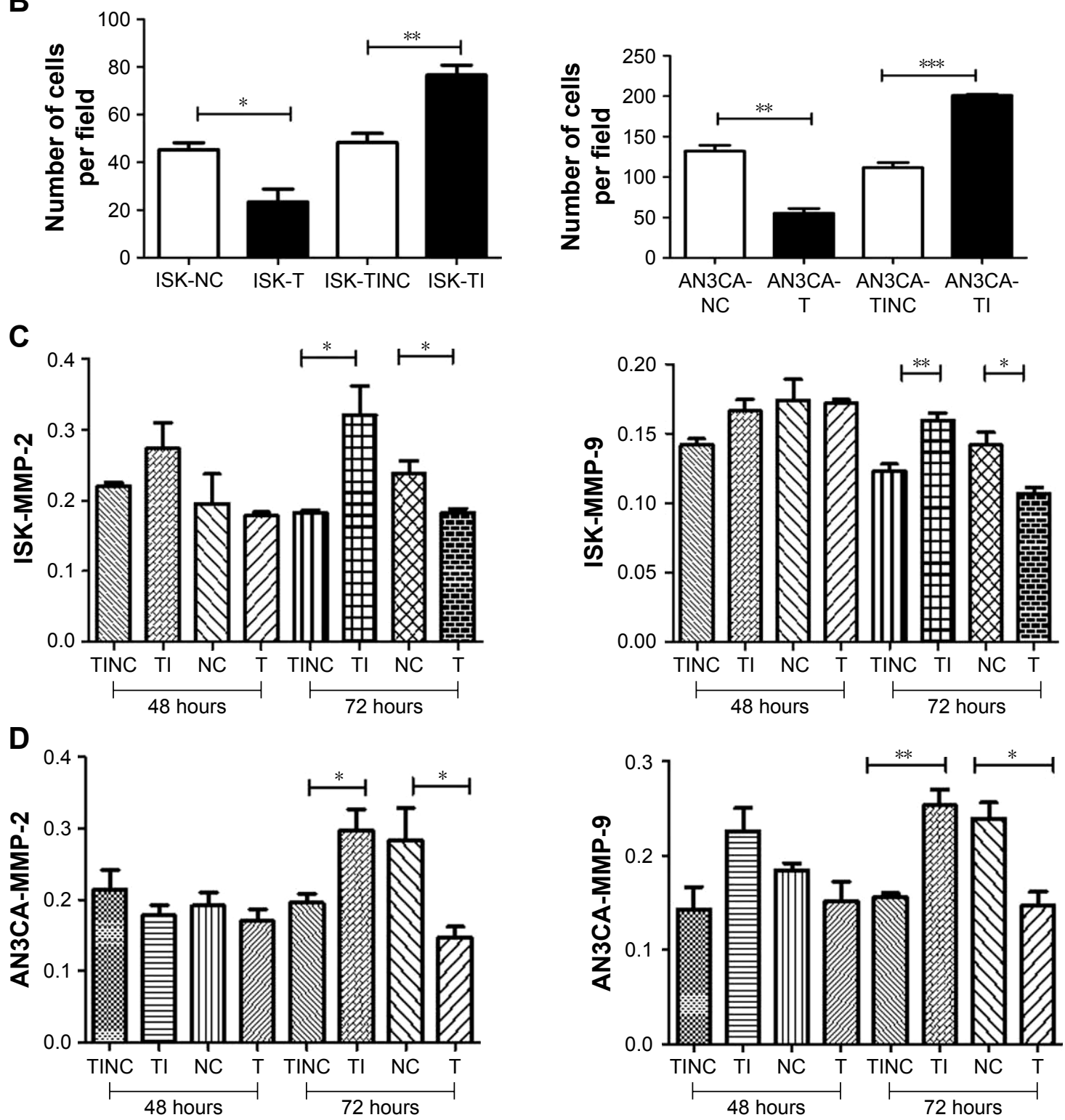

Figure 5 Cell migration was measured by a transwell migration assay.

Notes: (A) Transwell assays of ISK and AN3CA cells. (B) Quantification of transwell assays in ISK and AN3CA cells. (C) Expression levels of MMP-2 and MMP-9 in ISK cells. (D) Expression levels of MMP-2 and MMP-9 in AN3CA cells. ${ }^{*} P<0.05, * * P<0.01$, and $* * * P<0.001$, compared with the respective control groups by $t$-test. Data were obtained from three independent experiments.

Abbreviations: NC, TRIB3 overexpression negative control group; T, TRIB3 overexpression group; TI-I, TRIB3-shRNA plasmid I group; TI-2, TRIB3-shRNA plasmid 2 group; TINC, TRIB3-shRNA inhibitor negative control group. 


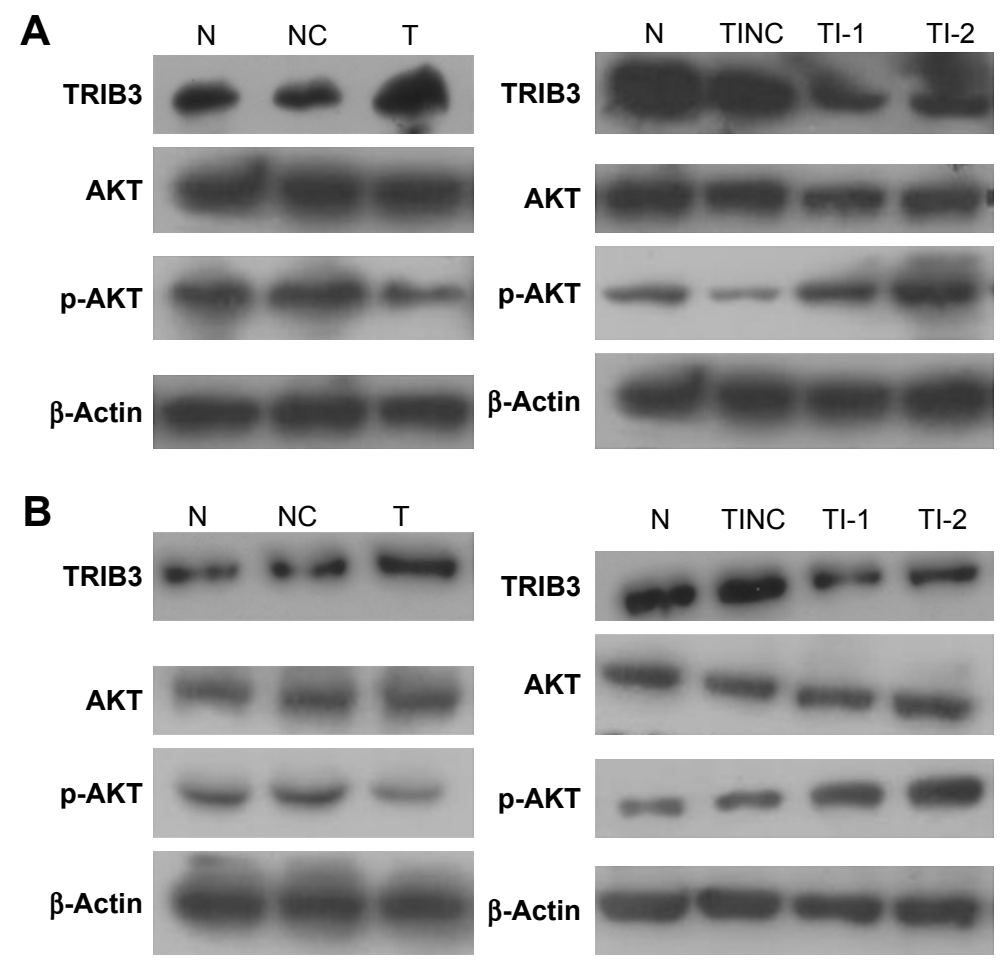

Figure 6 AKT and p-AKT levels in (A) ISK cells and (B) AN3CA cells were examined by Western blot. N, normal control group; NC: TRIB3 overexpression negative control group; T: TRIB3 overexpression group; TI: TRIB3 inhibitor group; TI-I: TRIB3-shRNA plasmid I group; TI-2: TRIB3-shRNA plasmid 2 group; TINC: TRIB3-shRNA inhibitor negative control group.

We found that TRIB3 was expressed at a relatively high level in endometritis, endometrial hyperplasia, and EC tissues compared to normal tissues. Furthermore, there was a significant statistical difference between EC tissues and normal tissues. Although fewer works have reported the alteration of TRIB3 expression in EC tissues, abundant investigations have suggested that TRIB3 expression levels were dramatically increased in cancer tissues when compared with the normal tissues. ${ }^{27,28}$ Additionally, TRIB3 has also been thought to play vital regulatory roles in cancer cell growth, proliferation, and apoptosis. ${ }^{28,29}$ A previous study demonstrated that upregulated TRIB3 suppressed endothelial cell growth and proliferation and was considered as critical in cell cycle regulation. ${ }^{30}$ However, no corresponding research has been undertaken in clarifying the effect of TRIB3 on common gynecological cancers such as EC. Here, the CCK-8 and plate clone formation assay revealed that TRIB 3 could inhibit the proliferation of ISK and AN3CA cells in vitro. Similarly, the high level of TRIB3 promoted cell apoptosis which was in line with the results of other cancer studies. ${ }^{31}$ Accordingly, we also found that the number of apoptotic EC cells was enhanced in the presence of TRIB3 overexpression, and conversely, the rate of apoptosis was remarkably decreased in EC cells transfected with shRNA. Nevertheless, the current results showed that TRIB3 functioned as a tumor suppressor (decreased proliferation and migration and increased apoptosis) but its expression was increased rather than decreased in malignant tissues compared to benign endometrium. This inconsistency in findings has also been found in other studies. A previous study demonstrated that TRIB3 was upregulated by ER stress and downregulated following genotoxic stress. ${ }^{32}$ Another investigation reported that whereas increased mRNA levels of TRIB3 were associated with a poor prognosis, increased TRIB3 protein level was related with a good prognosis in human breast cancer patients. ${ }^{33}$ These discrepancies may be caused by the fact that TRIB3 is involved in hypoxia tolerance and a number of regulatory feedback loops. ${ }^{33}$ Further studies are needed to elucidate the role of TRIB3 in EC progression.

It is well acknowledged that cell migration and invasion are distinctive characteristics of malignant tumors. Unfortunately, the regulatory mechanisms of TRIB3 in EC cell migration and invasion have not been elucidated. In this study, we focused on investigating the effect of TRIB3 on migratory and invasive ability of EC cells. The results showed that TRIB3 significantly inhibited migration and invasion of ISK and AN3CA cells compared with the control groups. Furthermore, accumulating evidence has implied that matrix metalloproteinases are closely correlated with the occurrence and progression of various cancers, especially cancer metastasis due to their involvement in the degradation of the 
extracellular matrix. ${ }^{34}$ Notably, MMP-2 and MMP-9 have been extensively investigated and regarded as prominent regulators that play pivotal roles in the migration and invasion of carcinoma. ${ }^{35,36}$ Therefore, we examined whether the expression levels of these two molecules were affected by the expression levels of TRIB3 in EC cell metastasis and the findings indicated that TRIB3 levels were negatively correlated with the expression levels of MMP-2 and MMP9. Additionally, there were significant statistical differences between TRIB3 experimental groups and control groups after 72 hours. These results suggested that TRIB3 inhibited EC migration and invasion probably through regulating the levels of MMP-2 and MMP-9.

Various reports have also proposed that TRIB3 could interact with AKT which participates in diverse cellular processes and is strongly associated with cancer development. ${ }^{37,38}$ Salazar et $\mathrm{al}^{39}$ argued that TRIB3 inhibited tumorigenesis primarily via controlling the phosphorylation of AKT. In this current research, we also observed that the upregulation of TRIB3 blocked the AKT phosphorylation in ISK and AN3CA cells and inhibition of TRIB3 by shRNA elevated the AKT phosphorylation levels and activated the AKT signaling pathway as shown by Western blot assay, which revealed that there was a significant inverse correlation between TRIB3 and AKT phosphorylation levels. More importantly, Martini et $\mathrm{a}^{40}$ stated that the activation of AKT signaling pathway blocked apoptosis and promoted the proliferation, migration, and invasion of cancer cells. Therefore, we speculated that TRIB3 enhanced apoptosis and suppressed proliferation and migration ability of EC cells probably through the inhibition of AKT signaling pathway.

This study also has several limitations which are worth noting. As shown in Figure 6A, there was a decrease in p-AKT level in the TRIB3-shRNA inhibitor negative control group when compared with normal ISK cell control group. We believe that this may have been caused by protein loading error, which should be checked by further experiments. Additionally, the association of TRIB3 expression levels and EC histological phases has not been determined in this study primarily due to the limited sample size of EC cells in phase I and phase II. Therefore, the relevant results still need to be confirmed with a larger sample in future. Moreover, the exhaustive molecular regulatory mechanisms still need to be elucidated due to the fact that TRIB3-mediated AKT signaling pathway involve complicated molecular components.

\section{Conclusion}

TRIB3 level in EC tissues was higher than control tissues and TRIB3 induced apoptosis and retarded proliferation, migration, and invasion of EC cells possibly by the inhibition of AKT signaling pathway. Thus, TRIB3 could serve as a novel therapeutic target for EC.

\section{Acknowledgments}

This study was supported by National Natural Science Foundation of China (No. 81502234) and Shanghai municipal medical and health discipline construction projects (No. 2017ZZ02015).

\section{Disclosure}

The authors report no conflicts of interest in this work.

\section{References}

1. Neacşu A, Marcu ML, Stănică CD, et al. Clinical and morphological correlations in early diagnosis of endometrial cancer. Rom J Morphol Embryol. 2018;59(2):527-531.

2. Ogane N, Hori SI, Yano M, et al. Preponderance of endometrial carcinoma in elderly patients. Mol Clin Oncol. 2018;9(3):269-273.

3. Iwase H, Furukawa S, Hirasawa T, et al. The clinical features of recurrent endometrial cancer in Japan: chemotherapy instead of radiotherapy as postoperative adjuvant treatment. Int J Gynecol Cancer. 2018;28(8): $1616-1623$.

4. Nakao K, Banno H, Kondo T, Hirabuki S, Sasaki H, Hoshiba T. Simultaneous resection of endometrial cancer and high-level paraaortic paraganglioma using retroperitoneoscopic surgery. Gynecol Oncol Rep. 2018;25:122-124.

5. Fialkova V, Vidomanova E, Balharek T, et al. DNA methylation as mechanism of apoptotic resistance development in endometrial cancer patients. Gen Physiol Biophys. 2017;36(05):521-529.

6. Rutkowski DT, Kaufman RJ. A trip to the ER: coping with stress. Trends Cell Biol. 2004;14(1):20-28.

7. Ulianich L, Insabato L. Endoplasmic reticulum stress in endometrial cancer. Front Med. 2014;1(1):5.

8. Ohoka N, Yoshii S, Hattori T, Onozaki K, Hayashi H. TRB3, a novel ER stress-inducible gene, is induced via ATF4-CHOP pathway and is involved in cell death. EMBO J. 2005;24(6):1243-1255.

9. Yan Q, Zhu H, Wang FH, et al. Inhibition of TRB3 protects photoreceptors against endoplasmic reticulum stress-induced apoptosis after experimental retinal detachment. Curr Eye Res. 2016;41(2):240-248.

10. Salazar M, Carracedo A, Salanueva IJ, et al. TRB3 links ER stress to autophagy in cannabinoid anti-tumoral action. Autophagy. 2009;5(7): 1048-1049.

11. Izrailit J, Berman HK, Datti A, Wrana JL, Reedijk M. High throughput kinase inhibitor screens reveal TRB3 and MAPK-ERK/TGF $\beta$ pathways as fundamental Notch regulators in breast cancer. Proc Natl Acad Sci US A. 2013;110(5):1714-1719.

12. Salazar M, Lorente M, García-Taboada E, et al. TRIB3 suppresses tumorigenesis by controlling mTORC2/AKT/FOXO signaling. Mol Cell Oncol. 2015;2(3):e980134.

13. Su J, Yan Y, Qu J, Xue X, Liu Z, Cai H. Emodin induces apoptosis of lung cancer cells through ER stress and the TRIB3/NF- $\kappa$ B pathway. Oncol Rep. 2017;37(3):1565-1572.

14. Zhou H, Luo Y, Chen JH, et al. Knockdown of TRB3 induces apoptosis in human lung adenocarcinoma cells through regulation of Notch 1 expression. Mol Med Rep. 2013;8(1):47-52. 
15. Toren P, Zoubeidi A. Targeting the PI3K/Akt pathway in prostate cancer: challenges and opportunities (review). Int J Oncol. 2014;45(5): 1793-1801.

16. Clark AR, Toker A. Signalling specificity in the Akt pathway in breast cancer. Biochem Soc Trans. 2014;42(5):1349-1355.

17. Restelli M, Magni M, Ruscica V, et al. A novel crosstalk between CCAR2 and Akt pathway in the regulation of cancer cell proliferation. Cell Death Dis. 2016;7(11):e2453.

18. Zhang J, Wen HJ, Guo ZM, et al. TRB3 overexpression due to endoplasmic reticulum stress inhibits Akt kinase activation of tongue squamous cell carcinoma. Oral Oncol. 2011;47(10):934-939.

19. Singh S, Asal R, Bhagat S. Multifunctional antioxidant nanoliposomemediated delivery of PTEN plasmids restore the expression of tumor suppressor protein and induce apoptosis in prostate cancer cells J Biomed Mater Res A. 2018;106(12):3152-3164.

20. Zhu D, Ye M, Zhang W. E6/E7 oncoproteins of high risk HPV-16 upregulate MT1-MMP, MMP-2 and MMP-9 and promote the migration of cervical cancer cells. Int J Clin Exp Pathol. 2015;8(5):4981-4989.

21. Suri V, Arora A. Management of endometrial cancer: a review. Rev Recent Clin Trials. 2015;10(4):309-316.

22. Dong P, Konno Y, Watari H, Hosaka M, Noguchi M, Sakuragi N. The impact of microRNA-mediated PI3K/Akt signaling on epithelialmesenchymal transition and cancer stemness in endometrial cancer. J Transl Med. 2014;12:231.

23. Dasari VR, Mazack V, Feng W, Nash J, Carey DJ, Gogoi R. Verteporfin exhibits YAP-independent anti-proliferative and cytotoxic effects in endometrial cancer cells. Oncotarget. 2017;8(17):28628-28640.

24. Hrstka R, Podhorec J, Nenutil R, et al. Tamoxifen-dependent induction of $A G R 2$ is associated with increased aggressiveness of endometrial cancer cells. Cancer Invest. 2017;35(5):313-324.

25. Fabi F, Adam $P$, Vincent $K$, et al. Inhibition of CRM1 activity sensitizes endometrial and ovarian cell lines to TRAIL-induced cell death. Cell Commun Signal. 2018;16(1):39.

26. Wennemers M, Bussink J, van den Beucken T, Sweep FC, Span PN Regulation of TRIB3 mRNA and protein in breast cancer. PLoS One. 2012;7(11):e49439.

27. Dong S, Xia J, Wang H, et al. Overexpression of TRIB3 promotes angiogenesis in human gastric cancer. Oncol Rep. 2016;36(4):2339-2348.

28. Miyoshi N, Ishii H, Mimori K, et al. Abnormal expression of TRIB3 in colorectal cancer: a novel marker for prognosis. Br J Cancer. 2009; 101(10):1664-1670.
29. Wu IJ, Lin RJ, Wang HC, Yuan TM, Chuang SM. TRIB3 downregulation enhances doxorubicin-induced cytotoxicity in gastric cancer cells. Arch Biochem Biophys. 2017;622:26-35.

30. Zou T, Liu WJ, Li SD, Zhou W, Yang JF, Zou CG. TRB3 mediates homocysteine-induced inhibition of endothelial cell proliferation. J Cell Physiol. 2011;226(11):2782-2789.

31. Ding C-Z, Guo X-F, Wang G-L, et al. High glucose contributes to the proliferation and migration of non-small-cell lung cancer cells via GAS5-TRIB3 axis. Biosci Rep. 2018;38(2):BSR20171014.

32. Corcoran CA, Luo X, He Q, Jiang C, Huang Y, Sheikh MS. Genotoxic and endoplasmic reticulum stresses differentially regulate TRB3 expression. Cancer Biol Ther. 2005;4(10):1063-1067.

33. Wennemers M, Bussink J, Grebenchtchikov N, Sweep FC, Span PN. TRIB3 protein denotes a good prognosis in breast cancer patients and is associated with hypoxia sensitivity. Radiother Oncol. 2011;101(1): 198-202.

34. Akter H, Park M, Kwon O-S, Song EJ, Park W-S, Kang M-J. Activation of matrix metalloproteinase-9 (MMP-9) by neurotensin promotes cell invasion and migration through ERK pathway in gastric cancer. Tumor Biol. 2015;36(8):6053-6062.

35. Kim YM, Ku MJ, Son YJ, Yun JM, Kim SH, Lee SY. Anti-metastatic effect of cantharidin in A549 human lung cancer cells. Arch Pharm Res. 2013;36(4):479-484.

36. Groblewska M, Siewko M, Mroczko B, Szmitkowski M. The role of matrix metalloproteinases (MMPs) and their inhibitors (TIMPs) in the development of esophageal cancer. Folia Histochem Cytobiol. 2012;50(1):12-19.

37. Faes S, Dormond O. PI3K and Akt: Unfaithful partners in cancer. Int J Mol Sci. 2015;16(9):21138-21152.

38. Nicoletti-Carvalho JE, Nogueira TC, Gorjão R, et al. UPR-mediated TRIB3 expression correlates with reduced Akt phosphorylation and inability of interleukin 6 to overcome palmitate-induced apoptosis in RINm5F cells. J Endocrinol. 2010;206(2):183-193.

39. Salazar M, Lorente M, García-Taboada E, et al. Loss of tribbles pseudokinase-3 promotes Akt-driven tumorigenesis via FoxO inactivation. Cell Death Differ. 2015;22(1):131-144.

40. Martini M, de Santis MC, Braccini L, Gulluni F, Hirsch E. PI3K/Akt signaling pathway and cancer: an updated review. Ann Med. 2014; 46(6):372-383.
OncoTargets and Therapy

\section{Publish your work in this journal}

OncoTargets and Therapy is an international, peer-reviewed, open access journal focusing on the pathological basis of all cancers, potential targets for therapy and treatment protocols employed to improve the management of cancer patients. The journal also focuses on the impact of management programs and new therapeutic agents and protocols on

\section{Dovepress}

patient perspectives such as quality of life, adherence and satisfaction. The manuscript management system is completely online and includes a very quick and fair peer-review system, which is all easy to use. Visit http://www.dovepress.com/testimonials.php to read real quotes from published authors. 